1 Supporting Information

2

3

4

5

6

\title{
Atmospheric lead emissions from coal-fired power plants with different boilers and APCDs in Guizhou, Southwest China
}

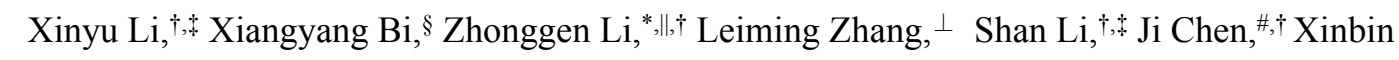 \\ Feng, ${ }^{*} \dagger,+\neq$ and Xuewu $\mathrm{Fu}^{\dagger}$
}

$\uparrow$ State Key Laboratory of Environmental Geochemistry, Institute of Geochemistry, Chinese Academy of Sciences, Guiyang 550081, China

$\$$ University of Chinese Academy of Sciences, Beijing 100049, China

$\S$ State Key Laboratory of Biogeology and Environmental Geology, School of Earth Sciences, China University of Geosciences, Wuhan 430074, China

॥ College of Resources and Environment, Zunyi Normal University, Zunyi 563006, China

${ }^{\perp}$ Air Quality Research Division, Science and Technology Branch, Environment and Climate Change Canada, Toronto, Canada

${ }^{*}$ College of Chemical Engineering, Huaqiao University, Xiamen 361021, China

\section{*Correspondence Author}

Email: lizhonggencn@126.com (Li Z.); Fengxinbin@mail.gyig.ac.cn (Feng X.)

Phone: +86-136-0851-7028; Fax: +86-851-2895-0875

\section{This supporting information document includes four Figures and six Tables.}




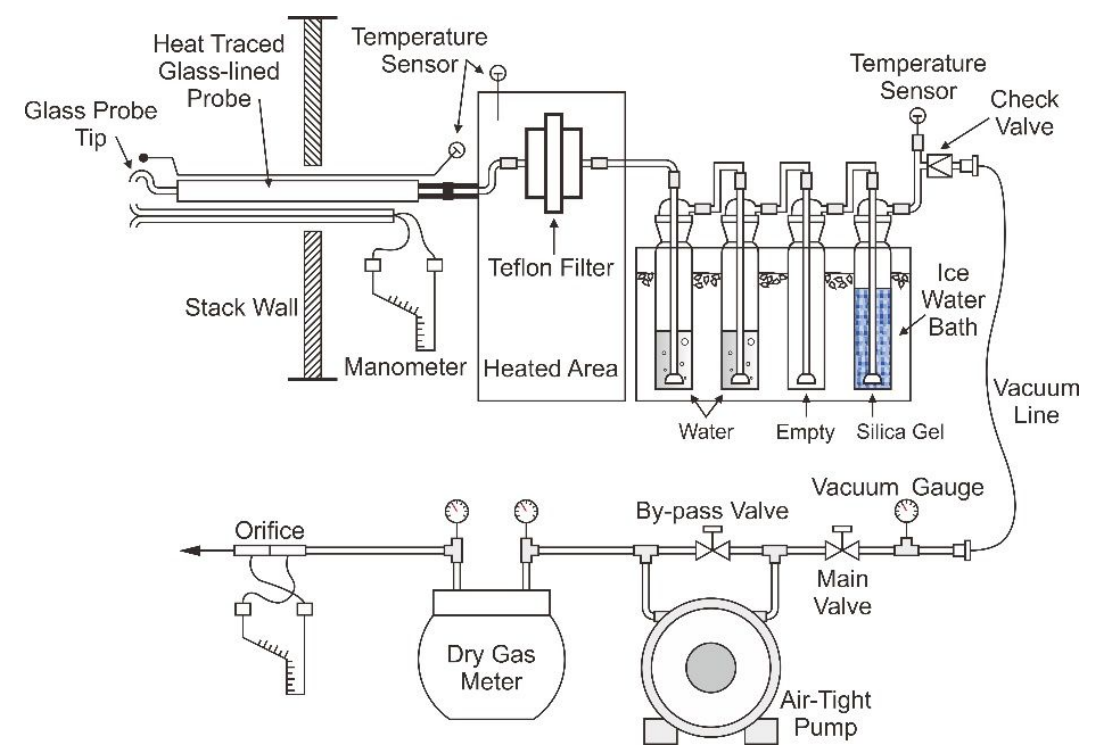

Figure S1. The U.S. EPA test method 5 for particulate matter sampling in the stack flue gas ${ }^{1}$ (U.S. EPA, 1996)
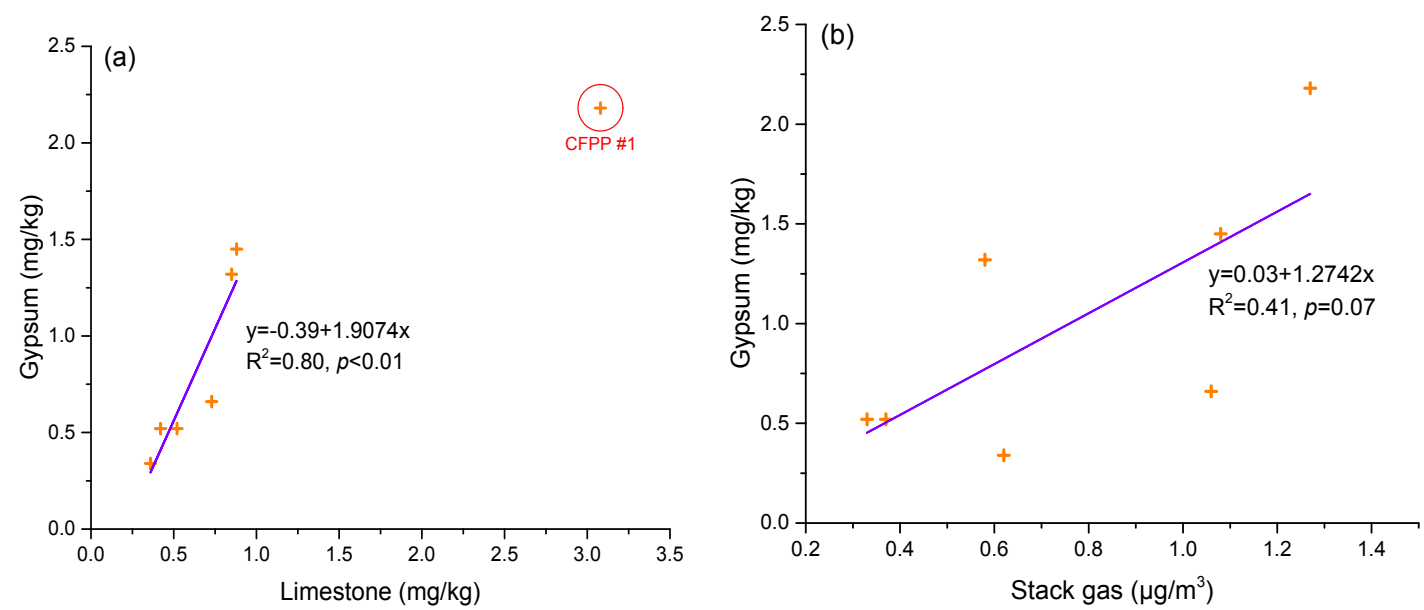

Figure S2. The correlation between $\mathrm{Pb}$ concentration in desulfurizing gypsum and (a) limestone, and 34

(b) stack gas of the eight tested CFPPs 


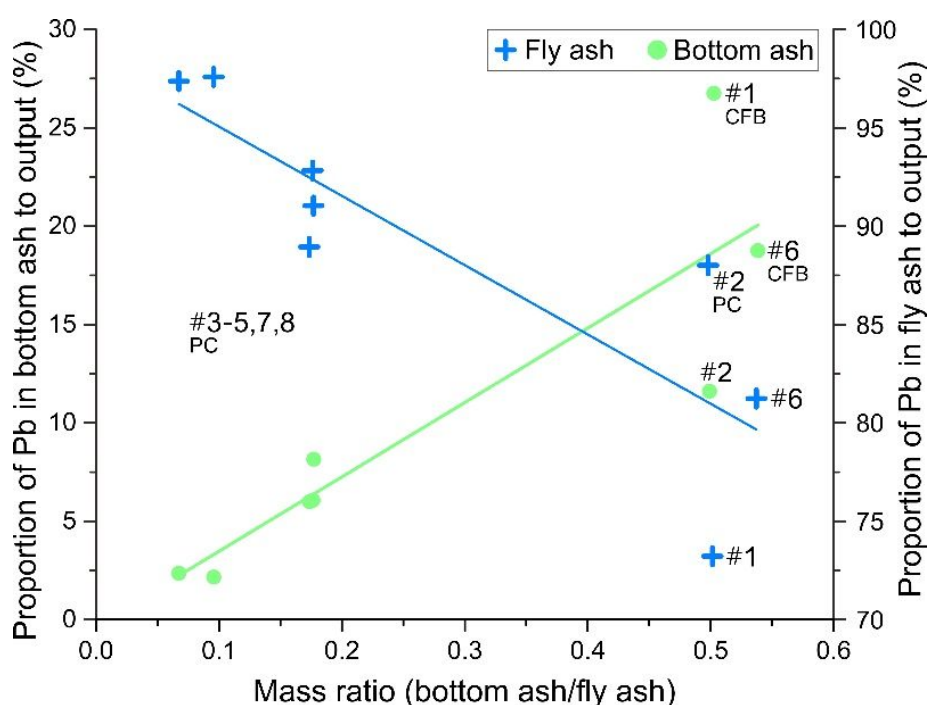

Figure S3. Trends of $\mathrm{Pb}$ proportion in CCPs to output with mass ratio (bottom ash/fly ash) in the tested CFPPs

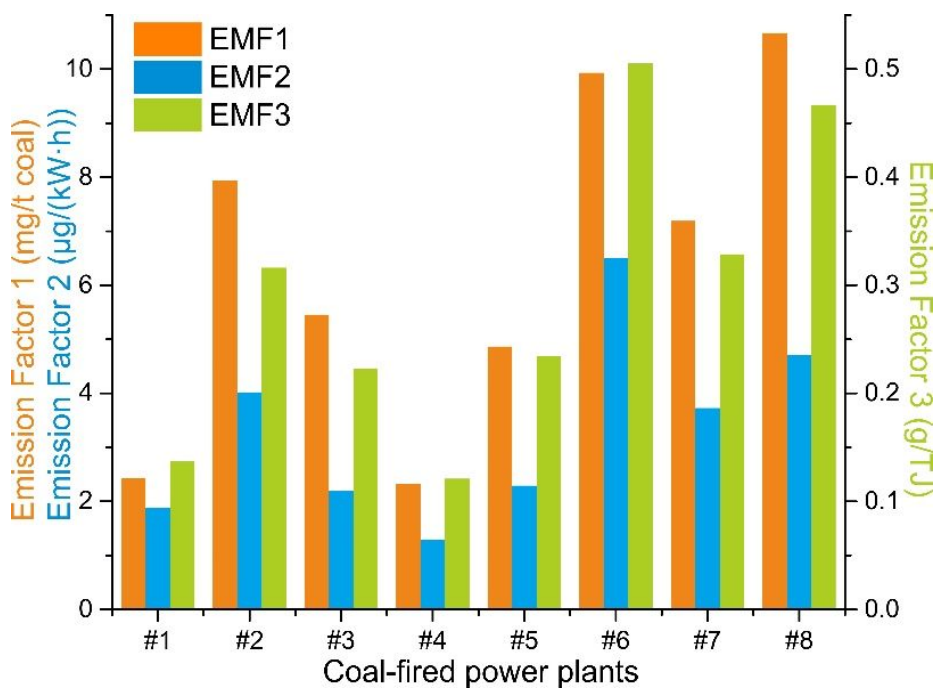

Figure S4. Pb emission factors (EMFs) of the eight tested CFPPs 
Table S1. The material consumption and production rate and PM content in the stack flue gas of the eight utility boiler system

\begin{tabular}{|c|c|c|c|c|c|c|c|c|}
\hline CFPPs & $\# 1$ & $\# 2$ & $\# 3$ & $\# 4$ & $\# 5$ & $\# 6$ & $\# 7$ & $\# 8$ \\
\hline \multirow[t]{2}{*}{ Feed fuel $(\mathrm{t} / \mathrm{d})$} & $1070^{\mathrm{a}}$ & 4376 & 3551 & 2032 & 5053 & 1730 & 2366 & 2776 \\
\hline & $2339^{b}$ & & & & & & & \\
\hline Limestone $(\mathrm{t} / \mathrm{d})$ & 36 & 269 & 140 & 108 & 448 & 384 & 265 & 393 \\
\hline Bottom ash $(\mathrm{t} / \mathrm{d})$ & 563 & 597 & 149 & 101 & 139 & 240 & 141 & 158 \\
\hline Fly ash $(\mathrm{t} / \mathrm{d})$ & 1120 & 1195 & 841 & 1054 & 2056 & 446 & 800 & 909 \\
\hline Gypsum (t/d) & 58 & 462 & 252 & 202 & 815 & l & 495 & 824 \\
\hline Stack flue gas $\left(10^{4} \mathrm{Nm}^{3} / \mathrm{d}\right)$ & 2213 & 3279 & 3367 & 1420 & 3995 & 1087 & 1571 & 2335 \\
\hline Actual operating power (MW) & 183 & 360 & 368 & 153 & 448 & 110 & 190 & 262.5 \\
\hline PM content in stack flue gas $\left(\mathrm{mg} / \mathrm{Nm}^{3}\right)$ & $\begin{array}{l}10.59 \pm 1.85 \\
(\text { No. }=4)\end{array}$ & $\begin{array}{l}18.28 \pm 0.30 \\
(\text { No. }=3)\end{array}$ & $\begin{array}{l}12.92 \pm 0.47 \\
(\text { No. }=3)\end{array}$ & $\begin{array}{l}10.15 \pm 3.80 \\
(\text { No. }=3)\end{array}$ & $\begin{array}{l}13.90 \pm 0.47 \\
(\text { No. }=3)\end{array}$ & $\begin{array}{l}31.26 \pm 9.12 \\
(\text { No. }=2)\end{array}$ & $\begin{array}{l}14.58 \pm 0.57 \\
(\text { No. }=2)\end{array}$ & $\begin{array}{l}36.42 \pm 0.35 \\
(\text { No. }=3)\end{array}$ \\
\hline
\end{tabular}

Table S2. Regional information of $\mathrm{Pb}$ concentrations in coal and coal ash

\begin{tabular}{lllll}
\hline Regions & Types & Coal $(\mathrm{mg} / \mathrm{kg})$ & Coal $\mathrm{ash}(\mathrm{mg} / \mathrm{kg})$ & References \\
\hline World & Brown coal & 6.6 & 38 & Ketris and Yudovich ${ }^{2}$ \\
World & Hard coal & 9 & 55 & Ketris and Yudovich $^{2}$ \\
World & Coal (Average) & 7.8 & 47 & Ketris and Yudovich $^{2}$ \\
China & Coal & 15.1 & $/$ & Dai et al. \\
Western Guizhou & Coal & 15 & $/$ & Dai et al. ${ }^{4}$ \\
\hline
\end{tabular}

Table S3. Relative enrichment factor (REF) of $\mathrm{Pb}$ in coal combustion productions (CCPs) of the eight CFPPs in the present study

\begin{tabular}{llllllllll}
\hline & Samples & $\# 1$ & $\# 2$ & $\# 3$ & $\# 4$ & $\# 5$ & $\# 6$ & $\# 7$ & $\# 8$ \\
\hline Relative enrichment factor & Bottom ash & 0.87 & 0.34 & 0.53 & 0.23 & 0.42 & 0.45 & 0.33 & 0.42 \\
(REF) & Fly ash & 1.20 & 1.28 & 1.04 & 0.99 & 1.17 & 1.06 & 0.90 & 1.09 \\
\hline
\end{tabular}


Table S4. Comparison of $\mathrm{Pb}$ concentrations in solid materials and $\mathrm{Pb}$ emissions data from coal-fired power plants (CFPPs)

\begin{tabular}{|c|c|c|c|c|c|c|c|c|c|c|}
\hline Region & Boiler type & APCDs & $\begin{array}{l}\text { Installed } \\
\text { capacity }\end{array}$ & $\begin{array}{l}\text { Coal } \\
(\mathrm{mg} / \mathrm{kg})\end{array}$ & $\begin{array}{l}\text { Bottom ash } \\
(\mathrm{mg} / \mathrm{kg})\end{array}$ & $\begin{array}{l}\text { Fly ash } \\
(\mathrm{mg} / \mathrm{kg})\end{array}$ & $\begin{array}{l}\text { Gypsum } \\
\text { (mg/kg) }\end{array}$ & $\begin{array}{l}\text { Stack flue gas } \\
\left(\mu \mathrm{g} / \mathrm{Nm}^{3}\right)\end{array}$ & Emission factors & References \\
\hline $\begin{array}{l}\text { Guizhou, China } \\
\text { (No. }=6 \text { ) }\end{array}$ & $\mathrm{PC}$ & SCR+C-ESP(-FF)+WFGD & $150-660 \mathrm{MW}$ & $16.49 \pm 6.69$ & $16.99 \pm 6.23$ & $48.00 \pm 13.78$ & $1.05 \pm 0.93$ & $0.82 \pm 0.33$ & $\begin{array}{l}6.41 \pm 2.62 \mathrm{mg} / \mathrm{t} \text { coal } \\
3.04 \pm 1.19 \mu \mathrm{g} /(\mathrm{kW} \cdot \mathrm{h}) \\
0.28 \pm 0.11 \mathrm{~g} / \mathrm{TJ}\end{array}$ & This study \\
\hline Guizhou, China & CFB & SNCR+C-ESP-FF+WFGD & $2 \times 300 \mathrm{MW}$ & $12.75-14.98$ & 26.41 & 36.3 & 0.52 & 0.37 & $\begin{array}{l}2.43 \mathrm{mg} / \mathrm{t} \text { coal } \\
1.88 \mu \mathrm{g} /(\mathrm{kW} \cdot \mathrm{h}) \\
0.14 \mathrm{~g} / \mathrm{TJ}\end{array}$ & This study \\
\hline Guizhou, China & CFB & IFD+ESP & $2 \times 150 \mathrm{MW}$ & 20.67 & 23.68 & 55.19 & / & 1.58 & $\begin{array}{l}9.93 \mathrm{mg} / \mathrm{t} \text { coal } \\
6.51 \mu \mathrm{g} /(\mathrm{kW} \cdot \mathrm{h}) \\
0.53 \mathrm{~g} / \mathrm{TJ}\end{array}$ & This study \\
\hline Jiangsu, China & $\mathrm{PC}$ & $\mathrm{SCR}+\mathrm{ESP}-\mathrm{FF}+\mathrm{WFGD}$ & $350 \mathrm{MW}$ & $2.94-8.56$ & $8.42-14.4$ & $76.7-79.4$ & $6.07-7.09$ & $0.18-0.26$ & $0.07-0.09 \mathrm{~g} / \mathrm{TJ}$ & Zhao et al. ${ }^{5}$ \\
\hline China & $\mathrm{PC}$ & $\mathrm{SCR}+\mathrm{ESP}+\mathrm{FGD}$ & $100 \mathrm{MW}$ & 23.02 & - & - & - & 1.42 & $11.53 \mathrm{mg} / \mathrm{t}$ coal & Zhao et al. ${ }^{6}$ \\
\hline Hebei, China & $\mathrm{PC}$ & $\mathrm{SCR}+\mathrm{ESP}-\mathrm{FF}+\mathrm{WFGD}$ & $660 \mathrm{MW}$ & 23.4 & 21.2 & 155.2 & 1.5 & 0.16 & $1.27 \mathrm{mg} / \mathrm{t}$ coal & Zhao et al. ${ }^{7}$ \\
\hline Guizhou, China & $\mathrm{PC}$ & C-ESP+WFGD & $300 \mathrm{MW}$ & 22.44 & 34.98 & 71.70 & 25.54 & 70.28 & - & Deng et al. ${ }^{8,9}$ \\
\hline Guizhou, China & CFB & C-ESP & $300 \mathrm{MW}$ & 34.90 & 25.26 & 92.08 & - & 154.61 & - & Deng et al. ${ }^{8,9}$ \\
\hline Shanghai, China & PC & $\mathrm{FF}+\mathrm{WFGD}$ & $300 \mathrm{MW}$ & 13.57 & 25.35 & 104.98 & 38.13 & 30.17 & - & Deng et al. ${ }^{8,9}$ \\
\hline Inner Mongolia, China & $\mathrm{PC}$ & $\mathrm{FF}+\mathrm{WFGD}$ & $300 \mathrm{MW}$ & 25.76 & 38.45 & 123.72 & 27.50 & 100.16 & - & Deng et al. ${ }^{8,9}$ \\
\hline Inner Mongolia, China & $\mathrm{PC}$ & C-ESP+WFGD & $600 \mathrm{MW}$ & 18.34 & 37.04 & 88.74 & 27.41 & 138.62 & - & Deng et al. ${ }^{8,9}$ \\
\hline Hubei, China & $\mathrm{PC}$ & $\mathrm{SCR}+\mathrm{C}-\mathrm{ESP}+\mathrm{WFGD}$ & $300 \mathrm{MW}$ & 58.50 & 13.27 & 154.01 & 16.67 & 320.15 & - & Deng et al. ${ }^{8,9}$ \\
\hline Anhui, China & $\mathrm{PC}$ & $\mathrm{ESP}+\mathrm{FGD}$ & - & $13.2-16.7$ & $15.3-17.2$ & $35.3-50.5$ & - & - & - & Tang et al. ${ }^{10}$ \\
\hline Anhui, China & $\mathrm{PC}$ & $\mathrm{ESP}+\mathrm{FGD}$ & - & 20.60 & $22.2-23.5$ & $62-113$ & - & - & - & Tang et al. ${ }^{10}$ \\
\hline China & $\mathrm{PC}$ & ESP & $220 \mathrm{t} \mathrm{coal} / \mathrm{h}$ & 16 & $7-21$ & $43-55$ & - & - & - & Huang et al. ${ }^{11}$ \\
\hline Shanxi, China & Unknown & Unknown & Unknown & 12.95 & 17.22 & 33.13 & - & 297 & - & Wang et al. ${ }^{12}$ \\
\hline China $($ No. $=30)$ & Unknown & $(\mathrm{SCR})+\mathrm{ESP} / \mathrm{FF}+\mathrm{WFGD}$ & 12-1000 MW & $8.50 \pm 5.68$ & - & - & - & $8.15 \pm 10.68$ & $64.34 \pm 81.21 \mathrm{mg} / \mathrm{t} \mathrm{coal}$ & Pei ${ }^{13}$ \\
\hline U.S. & $\mathrm{PC}$ & $\mathrm{SCR}+\mathrm{ESP}+\mathrm{WFGD}$ & Unknown & 53.00 & 15.00 & 41.80 & - & - & - & Swanson et al. ${ }^{14}$ \\
\hline U.S. & $\mathrm{PC}$ & FF-ESP+FGD & Unknown & 46.80 & 19.20 & 43.10 & - & - & - & Swanson et al. ${ }^{14}$ \\
\hline Western India & Unknown & ESP & $220 \mathrm{MW}$ & $29.4 \pm 8.6$ & $325.6 \pm 21.7$ & $266.1 \pm 18.9$ & - & $1.3 \pm 0.8$ & $0.04-0.06 \mathrm{~g} / \mathrm{TJ}$ & Reddy et al. ${ }^{15}$ \\
\hline Chhattisgarh, India & Unknown & Unknown & $150 \mathrm{MW}$ & 10.42 & 14.24 & 22.93 & - & - & - & Bhangare et al. ${ }^{16}$ \\
\hline Orrisa, India & Unknown & Unknown & $3000 \mathrm{MW}$ & 20.37 & 28.28 & 35.30 & - & - & - & Bhangare et al. ${ }^{16}$ \\
\hline Andhra Pradesh, India & Unknown & Unknown & $1000 \mathrm{MW}$ & 5.03 & 10.50 & 27.70 & - & - & - & Bhangare et al. ${ }^{16}$ \\
\hline Madhya Pradesh, India & Unknown & Unknown & $2250 \mathrm{MW}$ & 3.25 & 8.80 & 7.60 & - & - & - & Bhangare et al. ${ }^{16}$ \\
\hline Andhra Pradesh, India & Unknown & Unknown & $90 \mathrm{MW}$ & 13.22 & 19.74 & 30.38 & - & - & - & Bhangare et al. ${ }^{16}$ \\
\hline Uttar Pradesh, India & Unknown & Unknown & $1050 \mathrm{MW}$ & 5.44 & 11.7 & 44.1 & - & - & - & Verma et al. ${ }^{17}$ \\
\hline Malaysia & Unknown & ESP+FGD & $700 \mathrm{MW}$ & 16.2 & $17.8-22.1$ & 63.7 & - & - & - & Mokhtar et al. ${ }^{18}$ \\
\hline Paraná, Brazil & Unknown & $\mathrm{FF}$ & $10 \mathrm{MW}$ & $50-107$ & $66-92$ & $258-627$ & - & - & - & Flues et al. ${ }^{19}$ \\
\hline
\end{tabular}


Table S5. Pb flow and mass balance of the eight tested utility boilers

59

\begin{tabular}{cllllllll}
\hline CFPPs & $\# 1$ & $\# 2$ & $\# 3$ & $\# 4$ & $\# 5$ & $\# 6$ & $\# 7$ & $\# 8$ \\
\hline Input (g/d) & 45852.46 & 62929.10 & 46462.07 & 31417.05 & 75776.25 & 36217.42 & 73427.22 & 29454.44 \\
Feed fuel & $16019.61^{\mathrm{a}}$ & 62732.03 & 46342.41 & 31371.84 & 75613.84 & 35755.51 & 73194.25 & 28244.70 \\
& $29814.05^{\mathrm{b}}$ & & & & & & & \\
Limestone & 18.80 & 197.08 & 119.66 & 45.20 & 162.41 & 461.91 & 232.97 & 1209.74 \\
Output (g/d) & 55570.89 & 78631.41 & 40909.00 & 36251.95 & 93492.82 & 30324.95 & 63068.81 & 35641.49 \\
Bottom ash & 14871.00 & 9126.95 & 3333.19 & 781.75 & 2201.99 & 5688.39 & 3824.88 & 2133.47 \\
Fly ash & 40660.82 & 69163.82 & 37223.25 & 35360.74 & 90988.05 & 24619.38 & 58510.34 & 31682.24 \\
Gypsum & 30.34 & 305.91 & 333.19 & 104.74 & 278.20 & $/$ & 716.58 & 1796.14 \\
Flue gas & 8.73 & 34.73 & 19.37 & 4.72 & 24.58 & 17.18 & 17.02 & 29.63 \\
Output/Input (\%) & 121.20 & 124.95 & 88.05 & 115.39 & 123.38 & 83.73 & 85.89 & 121.01 \\
\hline 60 & & & & & & &
\end{tabular}

$60 \quad$ a, Gangue; b, Coal slime

61

62

63 64 65

Table S6. Pb contributions from different input and output materials in the eight tested utility boilers

\begin{tabular}{llllllllll}
\hline Direction & Samples & $\# 1$ & $\# 2$ & $\# 3$ & $\# 4$ & $\# 5$ & $\# 6$ & $\# 7$ & $\# 8$ \\
\hline Input & Feed fuel & $\begin{array}{l}34.94 \%{ }^{\mathrm{a}} \\
(65.02 \% \mathrm{~b}\end{array}$ & $99.69 \%$ & $99.74 \%$ & $99.86 \%$ & $99.79 \%$ & $98.72 \%$ & $99.68 \%$ & $95.89 \%$ \\
& & & & & & & & \\
\multirow{5}{*}{ Output } & Limestone & $0.04 \%$ & $0.31 \%$ & $0.26 \%$ & $0.14 \%$ & $0.21 \%$ & $1.28 \%$ & $0.32 \%$ & $4.11 \%$ \\
& Bottom ash & $26.76 \%$ & $11.61 \%$ & $8.15 \%$ & $2.16 \%$ & $2.36 \%$ & $18.76 \%$ & $6.06 \%$ & $5.99 \%$ \\
& Fly ash & $73.17 \%$ & $87.96 \%$ & $90.99 \%$ & $97.54 \%$ & $97.32 \%$ & $81.19 \%$ & $92.77 \%$ & $88.89 \%$ \\
& Gypsum & $0.05 \%$ & $0.39 \%$ & $0.81 \%$ & $0.29 \%$ & $0.30 \%$ & $/$ & $1.14 \%$ & $5.04 \%$ \\
& Flue gas & $0.02 \%$ & $0.04 \%$ & $0.05 \%$ & $0.01 \%$ & $0.03 \%$ & $0.06 \%$ & $0.03 \%$ & $0.08 \%$ \\
\hline
\end{tabular}

66

a, Gangue; b, Coal slime 


\section{References}

(1) U.S. Environmental Protection Agency (U.S. EPA). Test Method 5. Determination of particulate matter emissions from stationary sources, 1996.

(2) Ketris, M. P.; Yudovich, Y. E. Estimations of Clarkes for carbonaceous biolithes: world averages for trace element contents in black shales and coals. Int. J. Coal Geol. 2009, 78, (2), 135-148.

(3) Dai, S.; Ren, D.; Chou, C.-L.; Finkelman, R. B.; Seredin, V. V.; Zhou, Y. Geochemistry of trace elements in Chinese coals: A review of abundances, genetic types, impacts on human health, and industrial utilization. Int. J. Coal Geol. 2012, 94, 3-21.

(4) Dai, S.; Ren, D.; Tang, Y.; Yue, M.; Hao, L. Concentration and distribution of elements in Late Permian coals from western Guizhou Province, China. Int. J. Coal Geol. 2005, 61, (1-2), 119-137.

(5) Zhao, S.; Duan, Y.; Chen, L.; Li, Y.; Yao, T.; Liu, S.; Liu, M.; Lu, J. Study on emission of hazardous trace elements in a $350 \mathrm{MW}$ coal-fired power plant. Part 2. arsenic, chromium, barium, manganese, lead. Environ. Pollut. 2017a, 226, 404-411.

(6) Zhao, S.; Duan, Y.; Li, C.; Li, Y.; Chen, C.; Liu, M.; Lu, J. Partitioning and emission of hazardous trace elements in a $100 \mathrm{MW}$ coal-fired power plant equipped with selective catalytic reduction, electrostatic precipitator, and wet flue gas desulfurization. Energy \& Fuels 2017b, 31, (11), 12383-12389.

(7) Zhao, S.; Duan, Y.; Tan, H.; Liu, M.; Wang, X.; Wu, L.; Wang, C.; Lv, J.; Yao, T.; She, M.; Tang, H. Migration and emission characteristics of trace elements in a $660 \mathrm{MW}$ coal-fired power plant of China. Energy \& Fuels 2016, 30, (7), 5937-5944.

(8) Deng, S.; Shi, Y.; Liu, Y.; Zhang, C.; Wang, X.; Cao, Q.; Li, S.; Zhang, F. Emission characteristics of $\mathrm{Cd}, \mathrm{Pb}$ and $\mathrm{Mn}$ from coal combustion: Field study at coal-fired power plants in China. Fuel Process. Technol. 2014, 126, 469-475.

(9) Deng, S.; Zhang, F.; Liu, Y.; Shi, Y.; Wang, H.; Zhang, C.; Wang, X.; Cao, Q. Lead emission and speciation of coal-fired power plants in China. China Environ. Sci. 2013, 33, 1199-1206 (In Chinese with English abstract).

(10) Tang, Q.; Liu, G.; Zhou, C.; Sun, R. Distribution of trace elements in feed coal and combustion residues from two coal-fired power plants at Huainan, Anhui, China. Fuel 2013, 107, 315-322.

(11) Huang, Y.; Jin, B.; Zhong, Z.; Xiao, R.; Tang, Z.; Ren, H. Trace elements (Mn, Cr, Pb, Se, $\mathrm{Zn}, \mathrm{Cd}$ and $\mathrm{Hg}$ ) in emissions from a pulverized coal boiler. Fuel Process. Technol. 2004, 86, (1), 23-32.

(12) Wang, W. F.; Qin, Y.; Song, D. Y. Study on the mobility and release of trace elements in coal-fired power plant. Acta Sci. Circumstantiae 2003, 23, 748-752 (In Chinese with English abstract). 
(13) Pei, B. Field measurement for lead emission in the plumes of coal-fired power plants. Acta Sci. Circumstantiae 2013, 33, 1697-1702 (In Chinese with English abstract). (14) Swanson, S. M.; Engle, M. A.; Ruppert, L. F.; Affolter, R. H.; Jones, K. B. Partitioning of selected trace elements in coal combustion products from two coal-burning power plants in the United States. Int. J. Coal Geol. 2013, 113, 116-126.

(15) Reddy, M. S.; Basha, S.; Joshi, H. V.; Jha, B. Evaluation of the emission characteristics of trace metals from coal and fuel oil fired power plants and their fate during combustion. J. Hazard. Mater. 2005, 123, 242-9.

112 (16) Bhangare, R. C.; Ajmal, P. Y.; Sahu, S. K.; Pandit, G. G.; Puranik, V. D., Distribution of trace elements in coal and combustion residues from five thermal power plants in India. Int. J. Coal Geol. 2011, 86, 349-356.

115 (17) Verma, S. K.; Masto, R. E.; Gautam, S.; Choudhury, D. P.; Ram, L. C.; Maiti, S. K.; Maity,

116 S., Investigations on PAHs and trace elements in coal and its combustion residues from a power 117 plant. Fuel 2015, 162, 138-147.

118 (18) Mokhtar, M. M.; Taib, R. M.; Hassim, M. H., Understanding selected trace elements 119 behavior in a coal-fired power plant in Malaysia for assessment of abatement technologies. 120 Journal of the Air \& Waste Management Association 2014, 64, (8), 867-878.

121 (19) Flues, M.; Sato, I. M.; Scapin, M. A.; Cotrim, M. E. B.; Camargo, I. M. C. Toxic elements mobility in coal and ashes of Figueira coal power plant, Brazil. Fuel 2013, 103, 430-436. 